DOI:

10.1038/nrm2263

\section{$\Rightarrow$ PROTEIN EVOLUTION Tracing the evolution of structure and function}

The amino-acid-scale changes by which proteins evolve new functions are hard to trace. Now, however, evolutionary and structural biologists have retraced the substitutions by which the hormone-regulated glucocorticoid receptor (GR) evolved new structure, and thereby function, from its ancient ancestor.

Thornton and colleagues had previously shown that the genes that encode GR and mineralocorticoid receptor (MR) are descendants of an ancestral corticoid receptor (AncCR). They had also 'resurrected' AncCR by determining its maximum likelihood sequence from the sequences of 60 steroid receptors of extant organisms and then synthesized, expressed and functionally characterized AncCR. Whereas MR and AncCR are preferentially activated by the hormones aldosterone and deoxycorticosterone, GR is activated only by cortisol.

To trace the evolution of GR's ligand specificity, Thornton, Ortlund and co-workers crystallized AncCR and determined its structure. They then resurrected successive ancestral GRs and characterized them. The older AncGR1 had MR/AncCR-like specificity, whereas the more recent AncGR2 had GR-like specificity. Thus, the specificity of GR for cortisol was gained 420-440 million years ago, between AncGR1 and AncGR2.

Although 37 substitutions differentiate AncGR2 from AncGR1, only 5 are strictly conserved between modern GRs and MRs. When introduced into AncGR1, a pair of these - S106P and L111Q - switched the receptor's preference to cortisol, but not to the extent of modern GRs. Crystal structures revealed the mechanism for this epistatic interaction: S106P unwinds a helix and repositions residue 111 in the ligand pocket, bringing it close to a hydroxyl group unique to cortisol; L111Q then generates a new hydrogen bond to this hydroxyl, enhancing cortisol binding but destabilizing binding to other ligands.

The authors expected the other 3 conserved substitutions to render AncGR1 completely cortisol specific. Instead, these abolished all activity owing to destabilization of the structural network that positions the receptor's activation-function helix. Using ancestral crystal structures, the authors predicted that 2 other historical substitutions, N26T and Q105L, would alter the network such that the 3 destabilizing mutations could be tolerated. Indeed, when all 7 substitutions were introduced, AncGR1 had full GR-like specificity.

So, the evolution of GR depended on the occurrence of permissive mutations that allowed subsequent destabilizing mutations, ultimately generating new function. This study shows that conformational epistasis - whereby mutation $X$ repositions residue $Y$ in space, thereby affecting the functions that can be induced by mutation of residue $\mathrm{Y}-$ might be an important theme in structural and functional evolution. Furthermore, these findings suggest that conformational epistasis could become a useful approach in protein engineering strategies.

Asher Mullard 